fournal of Medical Genetics (1974). 11, 371.

\title{
Chromocentres in polymorphs as interphase markers for chromosomes having increased constitutive heterochromatin
}

\author{
N. B. ATKIN and MARION C. BAKER \\ Department of Cancer Research, Mount Vernon Hospital, Northwood, Middlesex HA6 2RN
}

\begin{abstract}
Summary. After staining with the BSG technique for constitutive heterochromatin, human polymorphs exhibit chromocentres which are presumably derived from these chromosomal regions. In five individuals having an extended centromeric heterochromatin region on one No. 1 chromosome, a particularly large chromocentre was seen in the majority of polymorphs. Thus, prominent chromocentres in polymorphs may indicate the presence of constitutive heterochromatin variants.
\end{abstract}

In man, variation in the form of an increased amount of constitutive heterochromatin in chromosome Nos. 1, 9, and 16 and the $\mathrm{Y}$ chromosome appears to be common (Chen and Ruddle, 1971; Craig-Holmes and Shaw, 1971; Lubs and Ruddle, 1971; Aula and Saksela, 1972; Alfi and Menon, 1973). The significance of this polymorphism is uncertain but in general it appears to be without phenotypic effect.

We have noted that in preparations subjected to the BSG technique for the demonstration of centromeric heterochromatin (Sumner, 1972) interphase nuclei commonly present a number of discrete chromocentres of varying size on a fairly homogeneous background. Whereas their incidence is somewhat variable in lymphocytes transformed by phytohaemagglutinin and in cancer cells they are more constant in their appearance in polymorphs. Four to eight chromocentres are commonly present; two of these are often slightly larger than the rest and may be derived from the No. 1 chromosomes which usually have the largest centromeric heterochromatin blocks (Fig. 1). In support of the view that these chromocentres correspond to the darkly-staining, juxta-centromeric regions seen in metaphase chromosomes with the same techique, we examined polymorphs in blood cultures from a patient with Hashimoto's disease known to have a

Received 29 April 1974. markedly extended heterochromatic region on one No. 1 chromosome: a particularly large chromocentre was seen (Fig. 2). Likewise, having noted the presence of a large chromocentre in polymorphs in smears made from ovarian carcinoma tissue, we were able to confirm that one No. 1 chromosome in cultured lymphocytes from this patient presented an extended heterochromatic region (Fig. 3). We have made similar observations on three further individuals. The large chromocentres were observed in over $50 \%$ of suitable polymorphs (ie, those that showed any chromocentres).

The chromocentres always appear to be situated in contact with the nuclear membrane. Drumsticks in the female, in keeping with their origin from the whole or greater part of an $\mathrm{X}$ chromosome, are generally lightly stained but may present a small peripheral chromocentre (Fig. 4). Smaller nuclear protrusions in both sexes (the 'small clubs' of Davidson and Robertson Smith, 1954) are, however, usually darkly stained and presumably encompass the constitutive heterochromatin regions of chromosomes.

Geraedts and Pearson (1973) noted the presence of large chromocentres due to variant No. 1 chromosomes in spermatozoa and lymphocytes, using a staining technique which stains especially intensely the centromeric heterochromatin of this chromosome. The technique we have used cannot distinguish between No. 1 and other chromosomes 


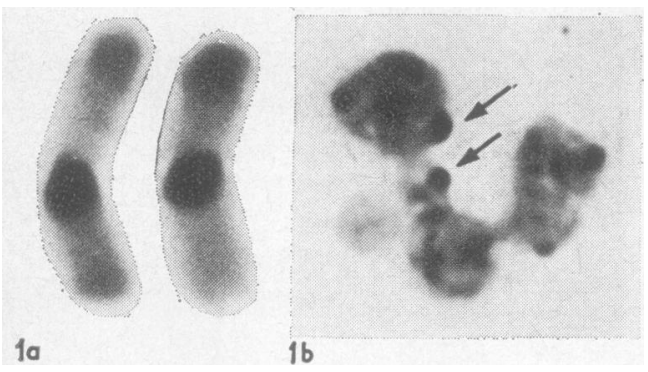

1a

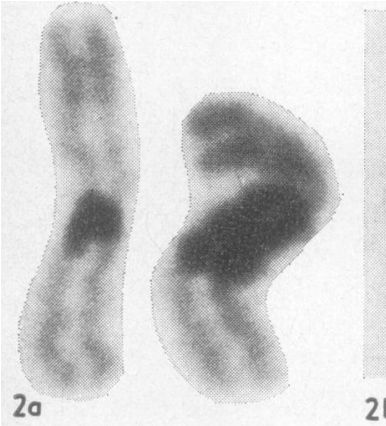

$2 a$

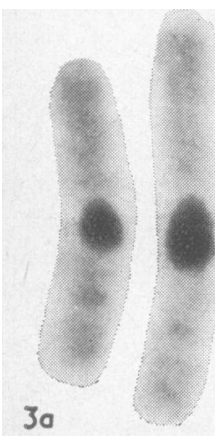

Fig. 1. Normal female: (a) No. 1 chromosomes $(\times 4610)$ and (b) polymorphs ( $\times 2420)$
Fig. 2. Female 'with Hashimoto's disease: (a) No. 1 chromosomes $(\times 4610),(b)$ and (c) polymorphs $(\times 2420)$.

Fig. 3. Patient with carcinoma of ovary: (a) No. 1 chromosomes $(\times 4610)$, (b) and (c) polymorphs $(\times 2420)$.

3c

Arrows indicate chromocentres possibly derived from the No. 1 chromosomes (Fig. 1) and the large chromocentre presumed to be derived from the No. 1 chromosome with an extended heterochromatic region (Figs. 2 and 3). All the chromosomes-and the polymorphs in Figs. $1 b, 2 b$, and $2 c-$ were from lymphocyte cultures. The polymorph in Fig. $3 b$ was from an air-dried preparation of carcinomaltissue fixed in methanol-acetic acid after hypotonic pretreatment, and that in Fig. $3 \mathrm{c}$ was from a blood smear fixed in methanol after air drying.

which may similarly have excessive heterochromatin; to determine which chromosome is involved, chromosome analysis would in general be necessary, although interphase chromocentres due to accentuated heterochromatin regions on No. 9 chromosomes may be specifically revealed by the 'Giemsa11' technique (Bobrow, Madan, and Pearson, 1972).

In the mouse, three or four heterochromatin blocks are present in polymorphs which are larger than those seen in man; this difference may be due to the 'aggregation of centromeres' of the telocentric chromosomes in the former species (Hsu et al, 1971) with the consequent tendency to fusion of the associated heterochromatic regions.

We conclude that examination of chromocentres in polymorphs in blood smears, following staining by a relatively simple $\mathrm{C}$-banding technique, may provide a useful screening procedure for the detection of constitutive heterochromatin variants. To some degree, the extent of the increase in heterochromatin can be assessed; thus, the approximately three-fold increase in one No. 1 chromosome of the 


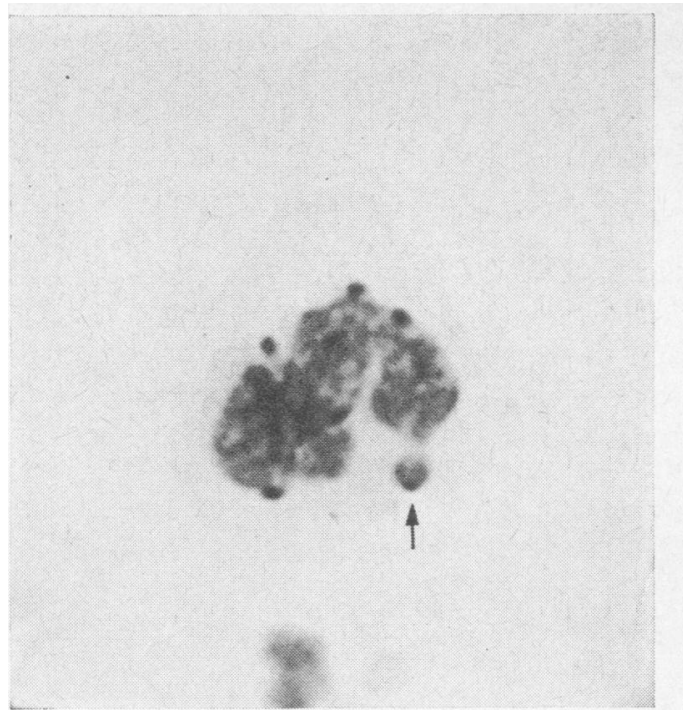

FIG. 4. Polymorph showing drumstick with small chromocentre (arrow) from normal female. ( $\times 1860$.) Blood smear fixed in methanol after air drying.

individual shown in Fig. 2 is associated with the presence of a somewhat larger chromocentre than is seen in Fig. 3, where the increase is two-fold or less.
We thank Miss K. Humphreys for technical assistance, $\mathrm{Mr}$ D. Astwood for preparing the photographs, and Mrs C. T. Elledge for secretarial assistance. This work was supported by a grant from the Cancer Research Campaign.

\section{REFERENCES}

Alfi, O. S. and Menon, R. (1973). A rapid C-band staining technique for chromosomes. Fournal of Laboratory and Clinical Medicine, 82, 692-694.

Aula, P. and Saksela, E. (1972). Banding characteristics of paracentric marker constrictions in human chromosomes. Hereditas Genetiskt Arkiv, 70, 309-310.

Bobrow, M., Madan, K., and Pearson, P. L. (1972). Staining of some specific regions of human chromosomes, particularly the secondary constriction of No. 9. Nature Nezv Biology, 238, 122124.

Chen, T. R. and Ruddle, F. H. (1971). Karyotype analysis utilizing differentially stained constitutive heterochromatin of human and murine chromosomes. Chromosoma, 34, 51-72.

Craig-Holmes, A. P. and Shaw, M. W. (1971). Polymorphism of human constitutive heterochromatin. Science, 174, 702-704.

Davidson, W. M. and Robertson Smith, D. (1954). A morphological sex difference in the polymorphonuclear neutrophil leucocytes. British Medical fournal, 2, 6-7.

Geraedts, J. and Pearson, P. (1973). Specific staining of the human no. 1 chromosome in spermatozoa. Humangenetik, 20, 171-173.

Hsu, T. C., Cooper, J. E. K., Mace, M. L., Jr., and Brinkley, B. R. (1971). Arrangement of centromeres in mouse cells. Chromosoma, 34, 73-87.

Lubs, H. A. and Ruddle, F. H. (1971). Chromosome polymorphism in American negro and white populations. Nature, 233, 134136.

Sumner, A. T. (1972). A simple technique for demonstrating centromeric heterochromatin. Experimental Cell Research, 75, 304306. 